\title{
Deep Circumscribed Morphea
}

National Cancer Institute

\section{Source}

National Cancer Institute. Deep Circumscribed Morphea. NCI Thesaurus. Code C121651.

Circumscribed morphea in which the lesions are found in the deep dermis, panniculus, fascia, or superficial muscle. 